The LISA Pathfinder DMU and Radiation Monitor

This article has been downloaded from IOPscience. Please scroll down to see the full text article.

2011 Class. Quantum Grav. 28094004

(http://iopscience.iop.org/0264-9381/28/9/094004)

View the table of contents for this issue, or go to the journal homepage for more

Download details:

IP Address: 147.83.119.168

The article was downloaded on 28/06/2011 at $11: 13$

Please note that terms and conditions apply. 


\title{
The LISA Pathfinder DMU and Radiation Monitor
}

\author{
P Canizares $^{1,2}$, M Chmeissani $^{3}$, A Conchillo $^{1,2}$, M Diaz-Aguiló $^{2,4}$, \\ E García-Berro ${ }^{2,4}$, L Gesa $^{1,2}$, F Gibert $^{2}$, C Grimani $^{5}$, I Lloro ${ }^{1,2}$, \\ A Lobo $^{1,2}$, I Mateos ${ }^{1,2}$, M Nofrarias ${ }^{6}$, J Ramos-Castro $^{7}, \mathbf{J ~ S a n j u a ́ n ~}^{8}$, \\ Carlos F Sopuerta $^{1,2}$, H M Araújo ${ }^{9}$ and P Wass ${ }^{9}$ \\ ${ }^{1}$ Institut de Ciències de l'Espai, CSIC, Facultat de Ciències, Torre C5 parell, 08193 Bellaterra, \\ Spain \\ ${ }^{2}$ Institut d'Estudis Espacials de Catalunya (IEEC), Edifici Nexus, Gran Capità 2-4, 08034 \\ Barcelona, Spain \\ ${ }^{3}$ Institut de Física d'Altes Energies (IFAE), Edifici CN, UAB Campus, 08193 Bellaterra, Spain \\ ${ }^{4}$ Departament de Física Aplicada, UPC, c/ Esteve Terrades 5, 08860 Castelldefels, Spain \\ ${ }^{5}$ Università degli Studi di Urbino, MFI Department, Via Santa Chiara 27, 61029 Urbino, and \\ INFN Florence, Italy \\ ${ }^{6}$ Max-Planck-Institut für Gravitationsphysik (Albert-Einstein-Institut), Callinstrasse 38, \\ D-30167 Hannover, Germany \\ ${ }^{7}$ Departament d'Enginyeria Electrònica, UPC, Campus Nord, Edifici C4, Jordi Girona 1-3, \\ 08034 Barcelona, Spain \\ ${ }^{8}$ Department of Physics, University of Florida, NPB-22258 PO Box 118 440, Gainesville, FL \\ 32611-8440, USA \\ ${ }^{9}$ High Energy Physics Blackett Laboratory, Prince Consort Road, Imperial College London, \\ London SW7 2BW, UK \\ E-mail: lobo@ieec.fcr.es
}

Received 28 September 2010, in final form 10 January 2011

Published 18 April 2011

Online at stacks.iop.org/CQG/28/094004

\begin{abstract}
The LISA Pathfinder DMU (Data Management Unit) flight model was formally accepted by ESA and ASD on 11 February 2010, after all hardware and software tests had been successfully completed. The diagnostics items are scheduled to be delivered by the end of 2010. In this paper, we review the requirements and performance of this instrumentation, specially focusing on the Radiation Monitor and the DMU, as well as the status of their programmed use during mission operations, on which work is ongoing at the time of writing.
\end{abstract}

PACS numbers: $04.80 . \mathrm{Nn}$, 95.55.Ym, 04.30.Nk, 07.87.+v, 07.60.Ly, 42.60.Mi

\section{Introduction}

LISA is a technologically sophisticated mission. It is so complex, in fact, that ESA decided to fly a precursor mission to ensure that technology readiness and maturity have reached a safe status to start LISA. Launch for Pathfinder is currently set for early 2013. 


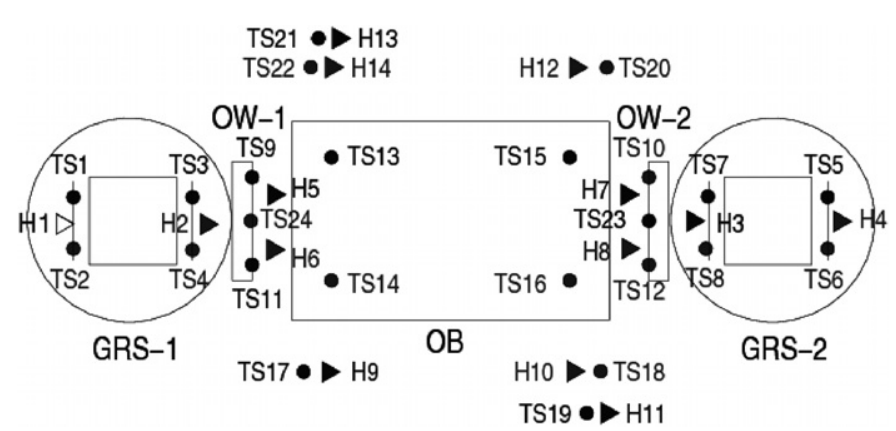

Figure 1. Schematics of the distribution of thermal diagnostics items, i.e. temperature sensors and precision heaters. Nomenclature: the triangles represent heaters and are labelled H1-H14; the circles represent temperature sensors and are labelled TS1-TS24; GRS-1 and GRS-2 are the gravitational reference sensors, OW-1 and OW-2 are the corresponding optical windows and OB is the optical bench. Heaters H9 through H14 and sensors, and TS17 through TS22 are attached to the LTP suspension struts-see more details of these structures in figure 3.

LISA Pathfinder (LPF) has a reduced acceleration noise budget, both in magnitude and in frequency band with respect to LISA [1]:

$$
S_{\delta a, \mathrm{LPF}}^{1 / 2}(\omega) \leqslant 3 \times 10^{-14}\left[1+\left(\frac{\omega / 2 \pi}{3 \mathrm{mHz}}\right)^{2}\right] \mathrm{ms}^{-2} \mathrm{~Hz}^{-1 / 2}
$$

in the frequency band between 1 and $30 \mathrm{mHz}$.

This noise can be apportioned to different components, each of them individually interfering with the mission performance. Requirements are set on each of those so that the total sum is compatible with equation (1) [1]. Thermal, magnetic and charged particle flux are specifically monitored by dedicated hardware and software, forming what is known as the data and diagnostics subsystem (DDS), which also includes the DMU (Data Management Unit), and the LTP (LISA Technology Package, or mission payload) computer. The DDS has been designed and built in IEEC-CSIC in Barcelona, Spain, and flight models of each of its parts have been delivered, or will be by the end of 2010, after passing extensive tests to ensure their performance during mission operations.

In this paper, we review the latest progress with the DDS parts, specially focusing on the Radiation Monitor (RM) and the DMU, which have been the subject of our work during the last few months. We will however give a quick summary of the other diagnostics items as well in the first sections; a more detailed description of them can be found in [2].

\section{Thermal diagnostics}

The thermal stability in the LTP core assembly (LCA) has to meet a very stringent requirement:

$$
S_{\delta T}^{1 / 2}(\omega) \leqslant 10^{-4} \mathrm{~K} \mathrm{~Hz}^{-1 / 2}, \quad 1 \mathrm{mHz} \leqslant \frac{\omega}{2 \pi} \leqslant 30 \mathrm{mHz} .
$$

A set of 24 temperature sensors is scattered around the LCA, figure 1, which is intended to measure temperatures at as many strategic spots. More specifically, in the outer walls of the electrode housings (EH) of both test masses (TM), at both optical windows (OW), at the optical bench (OB) and in the suspension struts (SS). In order to be able to make significant measurements in an environment with such a degree of thermal stability as required by 


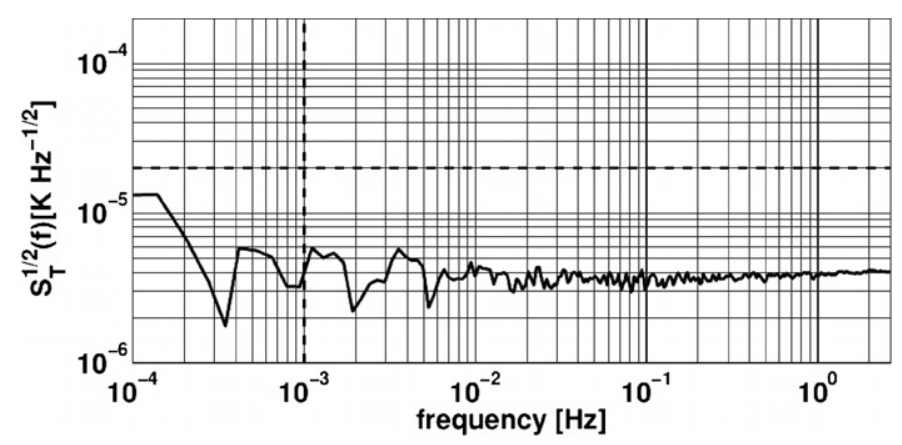

Figure 2. Flight model temperature sensor behaviour. The plot corresponds to a differential measurement between two NTCs. As can be seen, it is fully compliant with the requirement in equation (3).

equation (2), an even more demanding requirement must be set on the performance of these sensors. This is [3]

$$
S_{\delta T, \text { sensors }}^{1 / 2}(\omega) \leqslant 10^{-5} \mathrm{~K} \mathrm{~Hz}^{-1 / 2}, \quad 1 \mathrm{mHz} \leqslant \frac{\omega}{2 \pi} \leqslant 30 \mathrm{mHz}
$$

The temperature sensing in the DDS is done by means of NTC (negative temperature coefficient) thermistors, because they have more pronounced slopes in the temperatureresistance plane than other devices such as platinum resistors. In addition, an extremely quiet front-end electronics must be used to drive them and acquire data. This electronics is part of the DMU, and figure 2 shows the performance of a differential temperature measurement between two NTCs as recorded in the latest tests done with flight model sensors and DMU. This was April 2010. A detail must be clarified: because of mass and power budget limitations, not every one of the 24 thermistors can have its own electronic board; rather, they are multiplexed in six groups of four. While this makes the system globally compliant with those constraints, it introduces additional noise, as the real sampling frequency is divided by the multiplexing factor in each thermistor. This is however not four, as one would infer, but six because an additional differential temperature plus a control measurement is also multiplexed in each block. Therefore, the multiplexed system noise is higher by a factor of $6^{1 / 2} \simeq 2.4$ than shown in figure 2. Note, however, that we are still fully within requirements. Actually, the (individual) NTC noise is below $10^{-5} \mathrm{~K} \mathrm{~Hz}^{-1 / 2}$ all the way down to $10^{-5} \mathrm{~Hz}$, i.e. well within the LISA band. This means the current LTP thermal system can probably be transferred basically as is into LISA.

\subsection{Precision heaters}

It needs to be stressed that such marvellously working temperature sensors are not by themselves of much use for the mission. As explained in the previous section, we need these monitors to record temperature fluctuations which affect the performance of the LTP. But how do we link a (known) temperature fluctuation with an (unknown) TM shake or an interferometer phase jitter? Although this link can be modelled, it also has to be established in flight by direct measurement-since the model may be inaccurate.

To this effect, a set of 14 precision heaters is also installed in the LTP at some suitable spots [4]. Their role is to inject controlled thermal signals in the system such that they are strong enough to be clearly seen in the LTP readout, i.e. with a signal-to-noise ratio around 


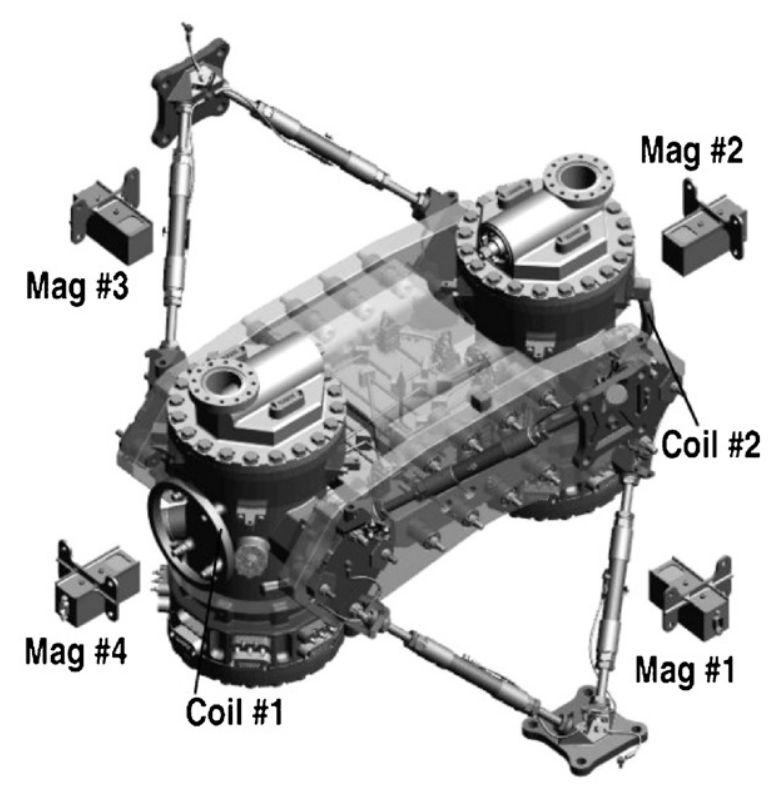

Figure 3. LTP diagnostic magnetometers and induction coils. For clarity, the former are shown levitating, but they are actually bolted to the surrounding cylindrical wall with the shown braces.

100. Temperature measurements are taken as well and hence a relationship is established between the latter and the observed response of the interferometer and/or the gravitational reference sensor (GRS). The analysis is done in frequency domain, so that a set of transfer functions is obtained relating temperature variations to LTP response. Finally, it is assumed that the behaviour of the LTP is maintained when the heaters are switched off.

Heaters are driven with very low noise DMU electronics. There are two major groups of heaters: those in the GRS and those elsewhere [4]. The ones in the second group are rather conventional kapton heaters, consisting in a wire winding held together by an elastic resin. These heaters, however, cannot be attached to the walls of the EH of the GRS due to their magnetic properties: they actually have some significant quantities of ferromagnetic elements, notably nickel, which are likely to compromise the stability of the TM. It was therefore decided that NTCs should also be used as heaters in that part of the LTP.

These NTCs are a bit more difficult to drive than the others due to their changing electric resistance upon being heated up. Indeed, when a voltage is applied to an NTC the current through it will heat it up; hence, its resistance will drop, and the current and power it dissipates will increase. It has been shown [5] that, under suitable conditions of initial NTC temperature, thermal resistance between the heater and the attachment block, and voltage range, this process ends up in a stationary state in a matter of about $10 \mathrm{~s}$. This is good enough for the time scales of the heating operations (normally a few thousand seconds), but the DMU has to be programmed so that these heaters be commanded with a voltage $V$ such that, in the stationary state, the power dissipated in the heater, $P_{\infty}$, is precisely the required one. The relationship between the voltage applied and the stationary state power dissipation turns out to be somewhat involved:

$$
P_{\infty}=\frac{V^{2}}{R\left(T_{\infty}\right)}=\frac{V^{2}}{R\left(T_{0}+\frac{\theta V^{2}}{R_{0}+\alpha_{0} \theta V^{2}}\right)}
$$


where $T_{0}$ is the NTC temperature just prior to activation (measured with an adjacent sensor), $\theta$ is the abovementioned thermal resistance $(\mathrm{K} / \mathrm{W})$ and $\alpha(T)=R^{-1} \mathrm{~d} R / \mathrm{d} T$ is the thermistor's temperature coefficient. The dependence of $R$ on $T$ is usually given by the Steinhart-Hart equation [6], again a somewhat involved mathematical expression. (In equation (4), $R_{0} \equiv$ $R\left(T_{0}\right)$ and $\alpha_{0} \equiv \alpha\left(T_{0}\right)$.)

The algebraic complexities of the above formulae make it impossible to invert equation (4) to obtain the voltage $V$ as a function of the power $P_{\infty}$ in a closed-form expression. This circumstance has motivated the use of look-up tables (LUT) in the DMU software which commands the GRS heaters: for a given thermal resistance $\theta$ (measured on ground) and an NTC temperature (measured dynamically on flight), the SW looks for the tabulated power which is closest to the one required for injection, then reads off the LUT the activation voltage, and applies it [7].

\section{Magnetic diagnostics}

The LTP TM are made of a $73 \% \mathrm{Au}+27 \%$ Pt alloy, a good combination to keep as low as possible their magnetic properties and, at the same time, to provide sufficient mechanical robustness to withstand launch shaking while caged. It is, however, impossible to avoid the presence of ferromagnetic residuals after the casting; hence, some magnetic remanence will be there. Limits have been set on the remnant magnetic moment $\mathbf{m}_{0}$ and susceptibility $\chi$ as [2]

$$
|\chi|<2.5 \times 10^{-5}, \quad\left|\mathbf{m}_{0}\right|<2 \times 10^{-8} \mathrm{Am}^{2} .
$$

These parameters couple to the surrounding magnetic field, mostly created by spacecraft electronic boxes and other components, such as solar panels, FEEP (field effect electric propulsion, the satellite's micro-thrusters), etc, thereby creating forces and torques on the TM, and fluctuations thereof, i.e. magnetic noise. According to standard electromagnetic theory, forces and torques are respectively given by

$$
\mathbf{F}=\left\langle\left[\left(\mathbf{M}+\frac{\chi}{\mu_{0}} \mathbf{B}\right) \cdot \nabla\right] \mathbf{B}\right\rangle V
$$

and

$$
\mathbf{N}=\left\langle\mathbf{M} \times \mathbf{B}+\mathbf{r} \times\left[(\mathbf{M} \cdot \nabla) \mathbf{B}+\frac{\chi}{\mu_{0}}(\mathbf{B} \cdot \nabla) \mathbf{B}\right]\right\rangle V .
$$

The meaning of symbols in the above formulae is as follows:

\begin{tabular}{ll}
\hline $\mathbf{B}$ & Magnetic induction field in the TM \\
\hline $\mathbf{M}$ & Density of magnetic moment (magnetization) of the TM \\
$\mathbf{r}$ & Vector distance to the TM centre of mass \\
$V$ & Volume of the TM \\
$\chi$ & Magnetic susceptibility of the TM \\
$\mu_{0}$ & Vacuum magnetic constant $\left(4 \pi \times 10^{-7} \mathrm{~m} \mathrm{~kg} \mathrm{~s}^{-2} \mathrm{~A}^{-2}\right)$ \\
\hline
\end{tabular}

while $\langle\cdots\rangle$ indicates TM volume average of the enclosed quantity. For example, for any magnitude $f(\mathbf{x})$, such average is defined by the volume integral

$$
\langle f\rangle \equiv \frac{1}{V} \int_{V} f(\mathbf{x}) \mathrm{d}^{3} x
$$




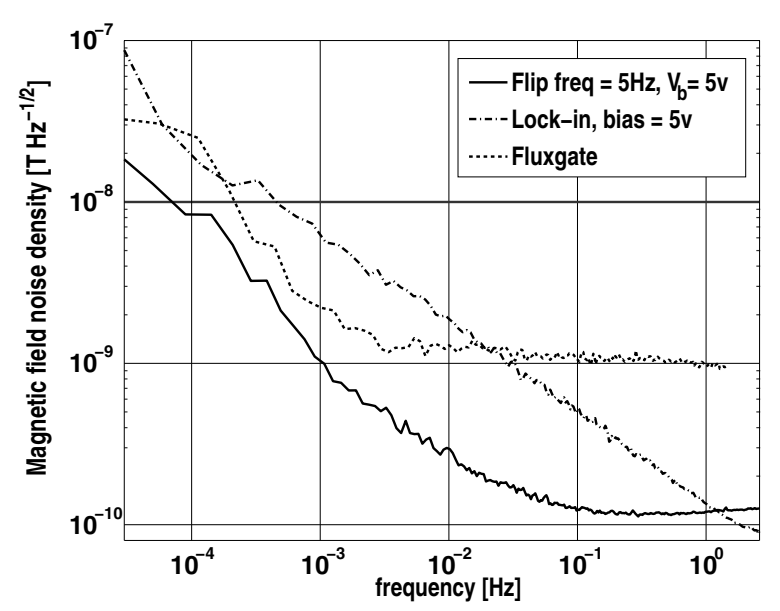

Figure 4. Magnetometer sensitivity curves. The horizontal, thicker grey line at $10^{-8}$ Tesla $\mathrm{Hz}^{-1 / 2}$ is the current LPF magnetometer noise requirement, which is comfortably met in all cases considered, i.e. for frequencies above $1 \mathrm{mHz}$. The curves show that the best performance corresponds to the AMR with a flip voltage scheme, showing significant improvements over the more conservative lock-in approach. The Fluxgate performs well but is slightly noisier at LISA frequencies.

over the TM volume. Magnetic noise can be readily inferred from the above formulae. There is no specific requirement on either magnetic field or gradient fluctuations; there is only one on the total contribution of magnetic disturbances to the overall mission acceleration noise budget; magnetic is required to stay below $40 \%$ of that budget [1]. On the other hand, there are specific requirements on the dc values of magnetic field and gradient, which should be kept below $10 \mu$ Tesla and $5 \mu$ Tesla $^{-1}$, respectively. This is because of the quadratic dependence of the force $\mathbf{F}$ on the magnetic field $\mathbf{B}$, due to the presence of a non-zero susceptibility-see equation (6); when it comes to evaluating the fluctuations, it is immediately seen that dc field values couple to gradient fluctuations, and also dc gradient values couple to field fluctuations.

Magnetic fields in the LTP are monitored by means of four tri-axial fluxgate magnetometers, located in the periphery of the LCA. These are not ideally suited to infer the magnetic field and gradient at the TMs, due to their distance to the latter. Classical interpolation methods have failed to produce acceptable results, but neural network algorithms have been developed and studied at IEEC which produce significant improvements in our ability to determine field values at the TMs [8]. The reader is also recommended to look into Aguiló's contribution to this Proceedings for more in-depth analysis and details.

\subsection{Alternative solutions}

While it is not possible to make changes now to the general LPF plan, e.g. magnetometers cannot be replaced, it would be unwise to think of LISA carrying on board a set of a few high performance but distant and voluminous fluxgate magnetometers. Ongoing research at IEEC has revealed that alternatives to the LPF scheme seem to exist. These are based on both a new type of sensors and driving electronics. The new sensors are tiny AMR (anisotropic magneto-resistor), while the electronics resorts to periodic flipping voltages to apply set-reset signals to the devices, thereby enhancing their performance. In figure 4 we see noise curves of various magnetic sensors and activation techniques which show the significant advantage 
which can be potentially drawn from the new AMRs. There are issues still to be properly addressed, perhaps most notably the possible magnetic back-action of the sensors, since they have tiny ferromagnetic cores. Analysis so far points towards such a negligible back-action; the reader will find much more detailed information in Mateos's contribution to this Conference Proceedings.

\subsection{Control coils}

Just like thermal diagnostics require precision heaters, magnetic diagnostics require precision induction coils. Their purpose is very similar to that of the heaters, though with a difference: strong magnetic signals injected by the induction coils, together with the LTP response to them (again in the order of SNR $\simeq 100$ ), can be used to determine the remnant magnetic moment and the susceptibility of the TMs. More information can be found in [2].

\section{The Radiation Monitor}

LPF will be stationed for operations in a Lissajous orbit around the Earth-Sun Lagrange point L1. This is about 1.5 million kilometres from Earth, i.e. well beyond its radiation belts. The spacecraft will thus be exposed to particles in the solar wind, but also to others, mostly of galactic origin. The composition of these fluxes in charged particles is roughly $90 \%$ protons, $8 \%$ helium ions and $2 \%$ heavier nuclei (carbon and higher $Z$ ) and electrons. These will charge the spacecraft through both direct and indirect deposition, since secondary particles are generated as the primary ones travel across the various materials. What creates concern about charging is actually only the TMs, as charge deposition in them generates spurious potentials; hence, noise in the GRS readout due to the random character of those depositions.

Extensive simulations done at Imperial College [9] with the public CERN tool Geant-4 show that only primary particles with energies roughly above $70 \mathrm{MeV}$ can possibly make it to the TM; lower energy ones are deterred by the satellite structure surrounding the TMs, and hence can cause no harm to the experiment. The simulations also show that the charging rate of the TMs depends on whether the primary particles are galactic cosmic rays (GCR) or correspond to flares in the Sun (SEP events, solar energetic particles ${ }^{10}$ ). These two types of flux have different energy spectra, and the way to distinguish between them is therefore to do spectroscopy. Figure 5 shows quite clearly the above distinction: spectra of GCR are displayed on the left and deposition rates in the right panel. Data are due to Araújo et al [10]. See also Grimani's contribution to this volume.

The RM role in LPF is to identify the charging rates in the TMs by short-term monitoring of the charged incoming particles. For this, a sensor consisting in two PIN diodes in telescopic configuration is used. The PINs are enclosed in a copper shielding which prevents primary particles with energies below $70 \mathrm{MeV}$ from reaching them, thereby recreating the actual situation in the TMs, see figure 6, left panel. An ionizing particle passing through the reverse-biased PIN junction will release many charge carriers ( 1 electron/hole pair per $3.6 \mathrm{eV}$ deposited) which is collected by a charge amplifier. The signal is then processed by a suitable electronics, which is able to (1) identify single-particle arrivals in the PIN, well above 6000 per second, the largest SEP shower ever observed, and (2) determine the energy deposition spectrum of an incoming particle flux [11]. Because the PINs are sensitive to photons, too, but these may not deposit charge in the TMs, the RM makes use of two PINs such that spectral information is only delivered when events are detected in coincidence in both PINs, thereby

${ }^{10}$ The solar wind contains mostly keV particles; only a relatively small fraction are above $100 \mathrm{MeV}$, and hence are named SEP. 

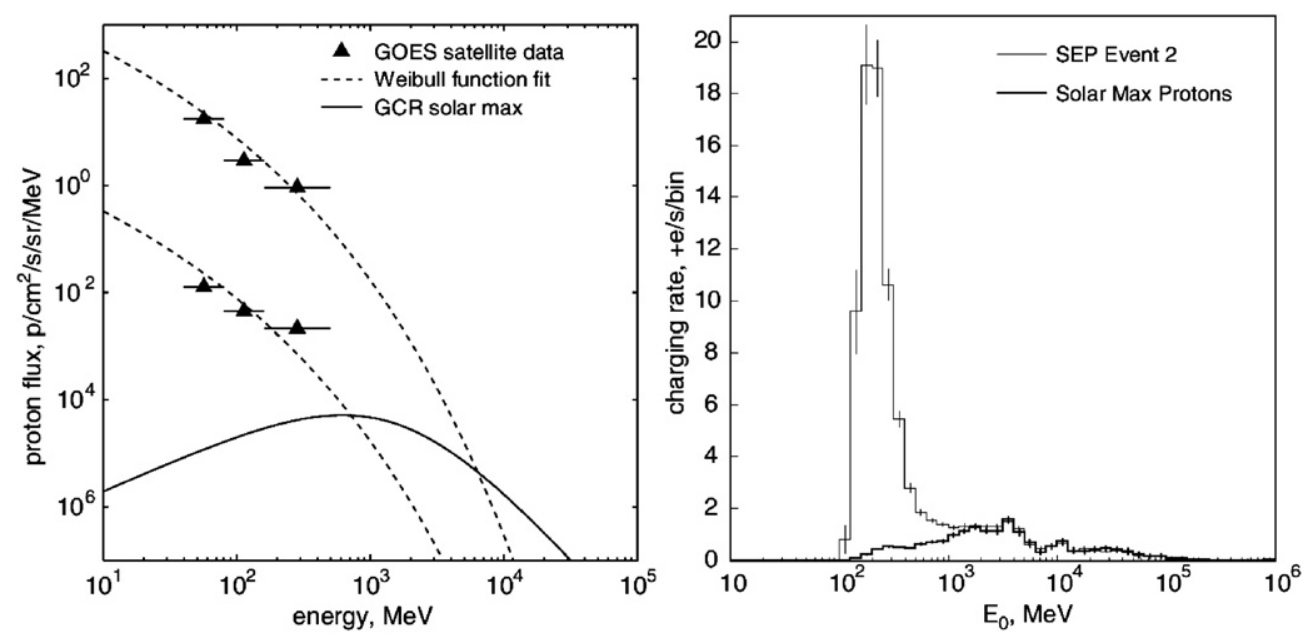

Figure 5. Left panel: spectra of primary energies in a giant SEP event (September 1989) and of a normal one (May 2001) as obtained from the GOES Observatory data, and fitted. Also shown is a GCR proton spectrum taken at solar maximum. Right panel: charging rates in the TMs for a 'normal' SEP event and GCR, also at solar maximum.

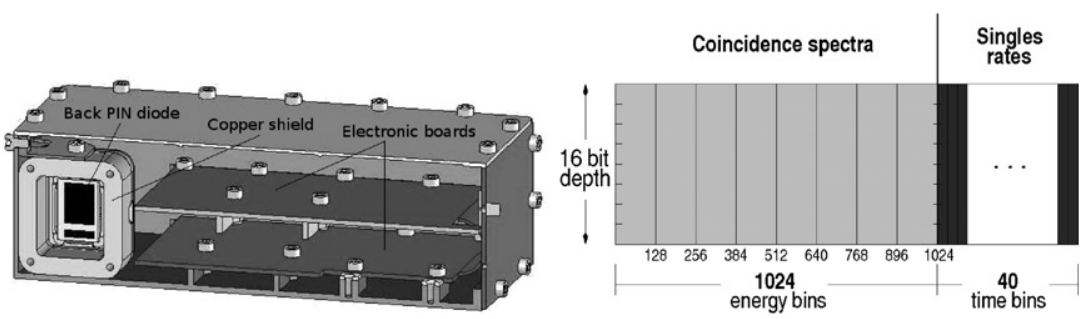

Figure 6. Left panel: schematics of the Radiation Monitor. Right panel: the Radiation Monitor data histogram, including 1024 energy bins for coincident events and 40 bins for singles countssee the text for more details.

filtering out photon events, which would only be seen in one, where it would be absorbed, hence never reaching the other. RM data are stored in a histogram format as shown in figure 6, right panel.

One such histogram is the RM telemetry unit. Bins are scanned and filled at $100 \mathrm{~Hz}$. Data are accumulated in the DMU during $600 \mathrm{~s}$ (i.e. $10 \mathrm{~min}$ ) and then sent to the on-board computer (OBC) to be telemetered to Earth according to mission schedules. Data are subsequently analysed off-line to look for correlations with the charge management system, which takes much longer periods to produce data. We can thus use the RM to detect shorter term charging fluctuations as well as modulations which should be useful to more thoroughly assess the whole process [12].

At the time of writing, the LPF RM flight model is a few weeks from final delivery. Many tests have been done on it before that: extensive electronics tests, magnetic moment measurements, vibration, temperature stability of the RM performance and proton irradiation. The latter was carried out at the Paul Scherrer Institute (Switzerland) where a proton beam facility was used to generate low dose fluxes and assess the RM response for various energies 


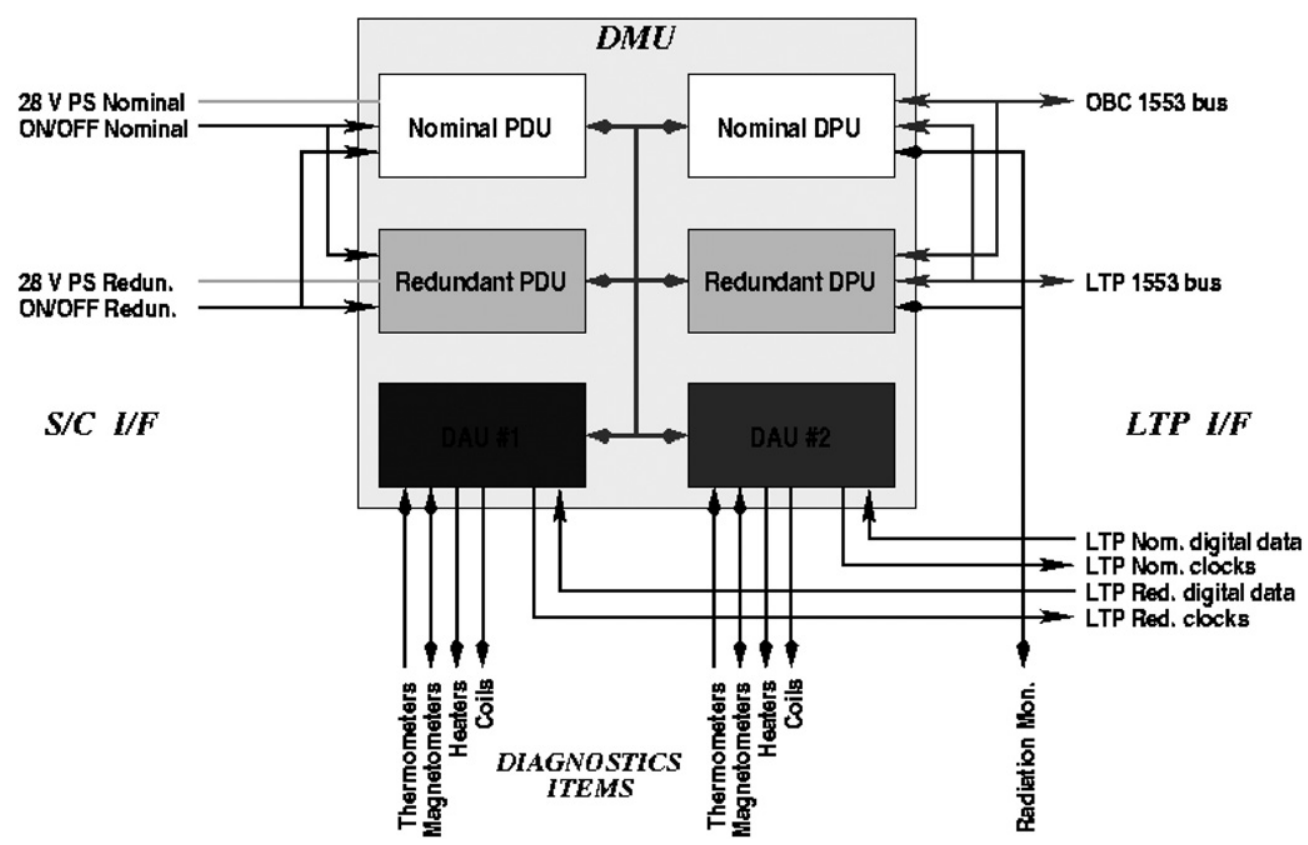

Figure 7. Schematics of the DMU boards and their LTP interfaces.

and beam incidence angles. The prototype was also submitted to such test, except it was much more aggressive at that time, as many more things had to be checked then. Now we are reassured that everything is in place and the test can be milder to avoid deterioration of the device before flying. Analysis of the irradiation results will soon be complete.

\section{The DMU}

The Data Management Unit (DMU) is the LTP computer, and has been designed, developed, manufactured and delivered by IEEC, Barcelona, on 11 February 2010. The DMU has three electronic boards: the Power Distribution Unit (PDU), the Data Acquisition Unit (DAU) and the Data Processing Unit (DPU), each of them duplicated (redundant) to cover potential failure in flight. Redundancy is however not full in the DAUs, where some diagnostics items are connected to only one DAU, see figure 7, again due to mass-energy quotas. Protection against possible DAU failure has been maximized by careful distribution of each DAU's tasks.

The DMU fully controls all the diagnostics item functionality, including activation, acquisition, and telemetry as described in sections 2 and 3, with the exception, however, of the RM, which internally acquires its data and communicates with the DMU via a serial RS 422 line. But the DMU has many more interfaces with other parts of the LTP, as shown in figure 7. Communication with the OBC and non-diagnostic parts of the LTP is done via two MIL-STD-1553 buses, respectively.

The DMU flight model hardware has undergone extensive testing before its formal acceptance by the Mission Architect.

- All diagnostics item performance/functional tests.

- Mechanical tests: centre of gravity, vibration, and pyrotechnic shock. 


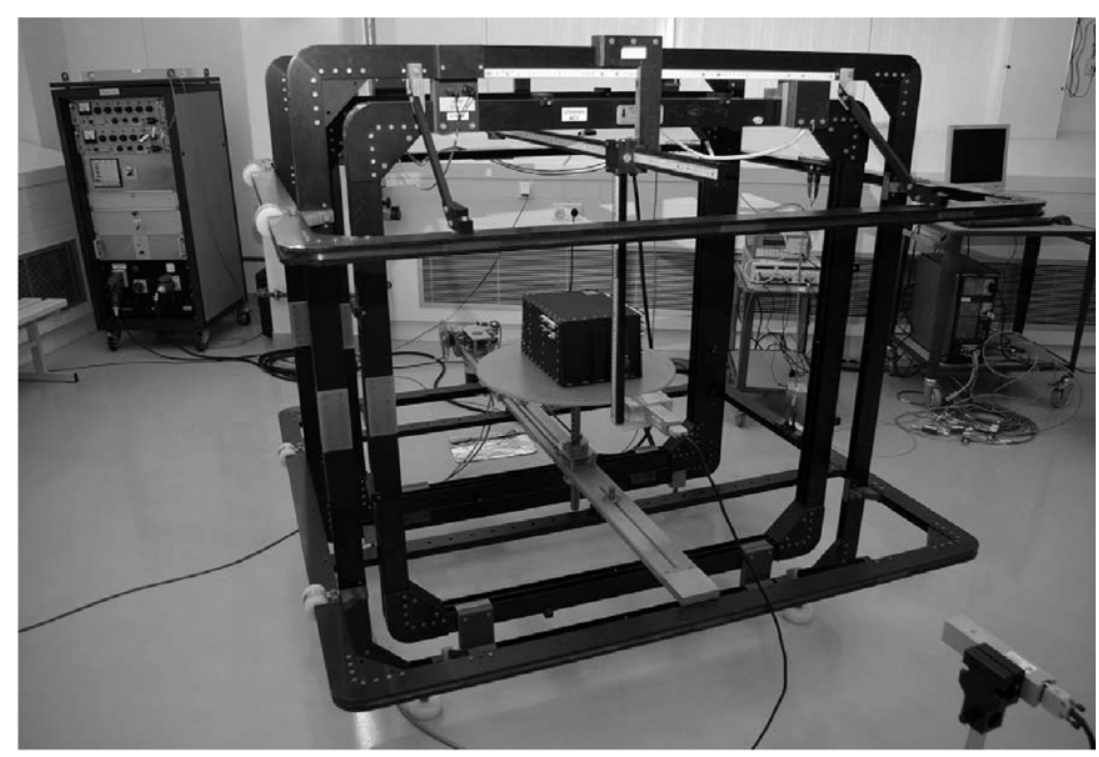

Figure 8. The DMU in a test facility at ESTEC. It is the dark box at the centre of a Helmholtz coil set, which shields the DMU from external disturbances during magnetic moment measurements.

- Thermal vacuum tests.

- Electromagnetic compatibility tests.

- Dipole magnetic moment measurements.

- Magnetic stability tests.

Some of them needed external facilities, such as magnetic tests done at ESTEC (see figure 8), and EMC tests at a local industrial company. All the above tests, however, have also been passed by the rest of the DDS hardware; they are indeed not specific to the DMU.

\subsection{The DMU software}

The DMU requires software to implement its functionality and to communicate with its various interfaces. This software has been created from scratch at IEEC, too, and has two main bodies: the boot software (BSW) and the application software (ASW).

The BSW is a minimal, non-real time operating system, which occupies 61 kilobytes of memory. It contains some 30000 lines of $C$ code, plus about 1000 of assembler code, and it was eventually burnt to a PROM (programmable read-only memory) chip, of course after extremely rigorous tests were passed, as the PROM cannot be either rewritten or replaced. The BSW starts the DMU and acts as scheduler, interrupt control and error detection and correction. A most important task for the mission which is also in charge of the BSW is to load the ASW, which understands and executes all the telecommands which are received from the mission operation centre (MOC) following the mission master plan. The BSW can also patch the ASW should there be an upgrade deemed necessary for the continuation of the mission operations. Figure 9, left, graphically summarizes these tasks. The BSW has long been burnt to an FM PROM, hence is fully ready for flight.

The ASW is built on top of RTEMS (real-time executive for multiprocessor systems), a real-time operating system, in order to cope with the hard real-time constraints (i.e. task 

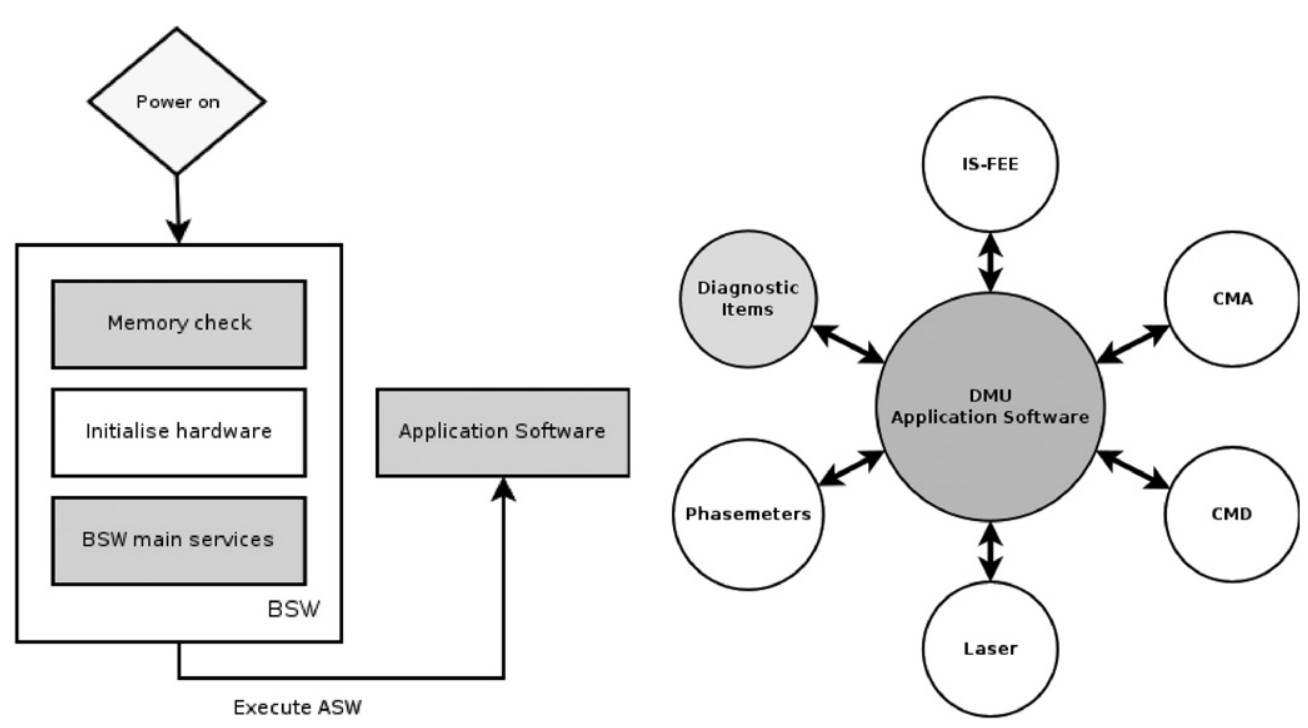

Figure 9. Conceptual diagram of the DMU software: BSW on the left and ASW on the right.

deadlines must be met on time) of the mission. The ASW, as already mentioned, manages all the scientific and technological functions of the DMU. It supports real time tasks up to a maximum frequency of $100 \mathrm{~Hz}$, and is encoded in some 70000 lines of $C$ code, plus 300 of assembler. In addition, it sends telemetry to the OBC via a MIL-STD-1553 bus. The ASW is stored in EEPROM (electrically erasable programmable read-only memory), which makes possible to receive upgrades from ground if necessary. It is executed from RAM. Stress tests done on the ASW show that it produces a maximum CPU load of $86 \%$, therefore a bit tight on margin. Work is in progress to bring down that ratio by a few percentile points.

The ASW tasks are graphically summarized in figure 9, right. In addition to diagnostics control, as already mentioned, it performs several operations and interfaces with other LTP subsystems. For example, it receives phasemeter data which analyses by single bin Fourier transform to produce feedback to the laser control system (e.g. frequency stabilisation). It also interacts with the caging mechanism, charge management device and gravitational reference sensors front-end electronics.

In addition to the mentioned stress tests, the ASW has undergone unit testing and validation, the latter by an independent entity which produced test scripts to check nominal test cases. Errors were filed and reported for debugging. The latest validated version is 2.3. ASW 2.4 was released in mid-August 2010, with all functionality in it. At the end of 2010 a validated and optimized version of ASW 2.4 will be ready.

The SW is a dynamic component of the DMU, and work on it is therefore envisaged all the way to mission completion.

\section{Conclusions}

We have briefly reviewed here the essential parts, as well as their functions, of the DDS. As shown, the Spanish hardware contribution to LPF is really complete, up to minor changes/details. The IEEC team is still active, and will be until the end of the mission. New tasks, not reported here, include the preparation of the mission operations, software 
(not to be confused with the DMU software) modelling of the system response to validate the PORs (payload operation requests) which constitute the basis for the entire mission Experiment Master Plan, and which are being prepared in the Science and Technology Operations Centre (STOC) in ESAC, near Madrid. In addition, on-line response and off-line data analysis of the mission yield have a lot to do with the diagnostics subsystem; hence, IEEC is very actively involved in these matters, too.

\section{Acknowledgments}

Financial support from the Spanish Ministry of Innovation and Research (MICINN), contract ESP2007-61712, is gratefully acknowledged, as well as AYA08-04211-C02-01. CFS acknowledges support from the Ramón y Cajal Programme of the same Ministry, and a Marie Curie International Reintegration Grant (MIRGCT-2007-205005/PHY) within the 7th European Community Framework Programme, and PC acknowledges an FPU PhD Grant from MICINN. Part of this work was also supported by AGAUR, Generalitat de Catalunya.

\section{References}

[1] Vitale S 2005 Science requirements and top-level architecture definition for the LISA Technology Package (LTP) on Board LISA Pathfinder (SMART-2) Report No LTPA-UTN-ScRD Iss003-Rev1

[2] Cañizares et al 2009 Class. Quantum Grav. 26094005

[3] Sanjuán J, Lobo A, Nofrarias M, Ramos-Castro J and Riu P 2007 Rev. Sci. Instrum. 78104904

[4] Lobo A 2010 LTP thermal response to periodic signals injected by heaters Report No S2 IEC-TN-3059

[5] Sanjuán J 2009 Development and validation of the thermal diagnostics instrumentation in LISA Pathfinder PhD Thesis Barcelona, appendix B

[6] Steinhart J S and Hart S R 1968 Calibration curves for thermistors Deep-Sea Res. 15497

[7] Conchillo A and Lobo A 2010 LTP electrode housing heaters commanding Report No S2 IEC-TN-3080

[8] Diaz-Aguiló M, García-Berro E and Lobo A 2010 Class. Quantum Grav. 27035005

[9] Wass P J, Araújo H M, Shaul D N A and Sumner T J 2005 Class. Quantum Grav. 22 S311

[10] Araújo H M et al 2005 Astropart. Phys. 22451

[11] Araújo H M and Wass P 2004 Note on the design of a particle monitor for LISA Pathfinder Technical Note S2 ICL-TN-3001, Imperial College, London

[12] Shaul D, Sumner T and Lobo A 2008 Analysis of data from the Radiation Monitor on LISA Pathfinder Report No S2 ICL-TN-3019 